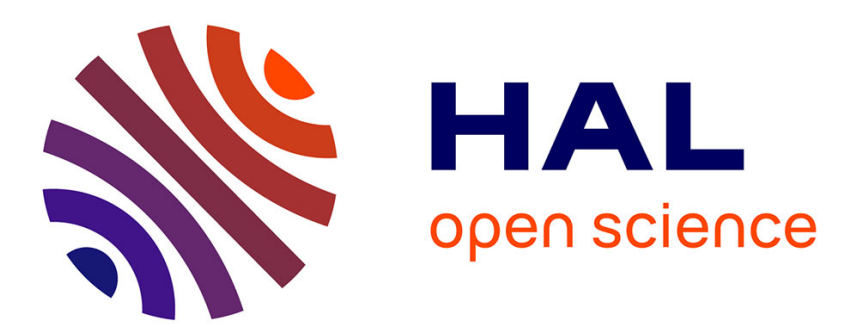

\title{
An emerging dynamics approach for synchronization of linear heterogeneous agents interconnected over switching topologies
}

Bikash Adhikari, Irinel-Constantin Morarescu, Elena Panteley

\section{To cite this version:}

Bikash Adhikari, Irinel-Constantin Morarescu, Elena Panteley. An emerging dynamics approach for synchronization of linear heterogeneous agents interconnected over switching topologies. IEEE Control Systems Letters, 2021, 5 (1), pp.43-48. 10.1109/LCSYS.2020.2999406 . hal-02940901

\section{HAL Id: hal-02940901 \\ https://hal.science/hal-02940901}

Submitted on 16 Sep 2020

HAL is a multi-disciplinary open access archive for the deposit and dissemination of scientific research documents, whether they are published or not. The documents may come from teaching and research institutions in France or abroad, or from public or private research centers.
L'archive ouverte pluridisciplinaire HAL, est destinée au dépôt et à la diffusion de documents scientifiques de niveau recherche, publiés ou non, émanant des établissements d'enseignement et de recherche français ou étrangers, des laboratoires publics ou privés. 


\title{
An emerging dynamics approach for synchronization of linear heterogeneous agents interconnected over switching topologies
}

\author{
B. Adhikari, I.-C. Morărescu, E. Panteley
}

\begin{abstract}
In this work, we propose an emergent dynamics based tool to approximate the synchronized behavior in large networks of interconnected linear systems with switching interconnection topologies and linear coupling. We assume that the networks are heterogeneous, but the states of all systems are of the same dimension. The goal of this paper is to show that, in the case of sufficiently large coupling gains, the network is practically synchronized, and its' synchronized behavior can be approximated by a reduced order switching system independent of the control gains. The results are ensured for strongly connected networks under fairly mild assumptions by introducing a minimum dwell-time between two consecutive switches. Numerical simulations illustrate the theoretical results.
\end{abstract}

\section{INTRODUCTION}

Due to its application in various disciplines such as physics, biology, economics, medicine, telecommunication, etc, synchronization got significant attention in the literature. Both networks with homogeneous/identical [7] and heterogeneous/nonidentical agent dynamics [5], [6], [16] have been considered with the objective to provide conditions that guarantee systems synchronization. Heterogeneous networked systems have more complex dynamic behavior and asymptotic synchronization may not be guaranteed, for example, for linear networked systems the internal model principle, [17] is necessary to ensure asymptotic synchronization. In this case a good trade-off is a practical synchronization [11], [13], which requires synchronization error to be ultimately bounded by arbitrarily small positive bounds. Other Lyapunov-based bounded synchronization results are provided in [10], [16].

Another difficulty that arises when dealing with complex dynamical network is related to the size of the network. Even if the network synchronizes, in many applications it is important to have an approximation of the network behaviour while avoiding simulation of the full system. Therefore, an important problem in large-scale networks analysis is the approximation of the dynamic behavior of the overall network by using a computationally tractable reduced order system. An effective method to develop such reduced order system uses the singular perturbation theory and is based on the time-scale separation and system analysis for interconnection gains tending to infinity. To the best of our knowledge, for the first time, a singular perturbations approach was

This work was purtially funded by CEFIPRA through the project "High performance formation control in the presence of uncertainties and communication constraints" and by ANR under grant HANDY ANR-18-CE40-0010

B. Adhikari and I.-C. Morărescu are with the Université de Lorraine, CNRS, CRAN, F-54000 Nancy, France (e-mails: firstname.name@univlorraine.fr). E. Panteley is with Laboratoire des Signaux et Systemes (L2S, CNRS - CentraleSupelec - Univ. Paris Sud), Gif-sur-Yvette, France. used for the analysis of networked systems in [2], where resulting reduced order system had an asymptotically stable equilibrium point. In [8], where a network of nonlinear oscillators was considered, the resulting reduced order model is an oscillator with asymptotically stable limit cycle. In [5], the authors use limiting equations to characterize the behavior of the reduced order system.

In the case of sheer size of the network, if the interconnection topologies are sparse and exhibit clusters [2], [12], [15], then the higher convergence speed within the clusters can be exploited to analyze the network behavior as shown in [2] for linear dynamics or in [1] for nonlinear ones. The time-scale separation can be used also in the case of time-varying topologies, as in [9], where the authors use a slowly varying weighted average of the clusters to define the slow variable of the overall network.

In this paper, we consider the case of heterogeneous linear systems with switching interconnections and we show that the synchronized behavior of the agents can be approximated by the emergent dynamics even if the topology switches. Precisely, using the approach presented in [8], it is shown that the emergent dynamics is a good approximation of the mean field dynamics as far as one uses a large enough gain in the decentralized feedback control law. Therefore, the main contribution of this work is twofold. First, we define the emergent dynamics in the more general case when the interconnection structure is not fixed. Second, we prove that this switching emergent dynamics approximates the overall behavior of the network dynamics with boundedness of the emergent dynamics as a sufficient condition. The proof is based on the notion of measure of a matrix as defined in [3]. The boundedness property is required to ensure the closeness of solutions for the mean-field dynamics of the network and the associated emergent dynamics.

The rest of the paper is organized as follows. Section II presents the problem formulation. The collective dynamics is presented and re-written in term of mean-field and error dynamics in Section III. The time scale separation between the two is highlighted in Section IV. In Section V, the approximation of the mean-field dynamics by the emergent dynamics is proven. This approximation holds under some mild conditions ensuring the stability of the fast dynamics and the boundedness of the emergent one. A numerical example illustrates our result before concluding.

Notation. In the sequel, $\mathbb{N}, \mathbb{R}_{+}, \mathbb{R}^{n}, \mathbb{R}^{n \times m}$ denote the set of natural numbers, non-negative real numbers, the space of real $n$-dimensional vectors and the set of all real matrices of 
dimension $n \times m$, respectively. The identity matrix of dimension $n \times n$ is represented as $I_{n}, \mathbf{1}_{N}$ is a column vector of ones and $\mathbf{0}_{n, m}$ is a matrix with dimension $n \times m$ whose all the components are zero. The symbol $\otimes$ denotes the Kronecker product and $\|\cdot\|$ is the 2-norm unless otherwise stated. The transpose and the inverse of a matrix $A$ are denoted as $A^{\top}$ and $A^{-1}$. We also use $x\left(t^{-}\right):=\lim _{\delta \rightarrow 0, \delta>0} x(t-\delta)$. We say function $\eta:(0, \infty) \rightarrow \mathbb{R}^{n}$ is $\mathcal{O}(\epsilon)$ iff there exists $c>0$, such that $\|\eta(t)\| \leq c \epsilon$.

\section{Problem Formulation}

Let us consider a network of $N$ heterogeneous linear systems called agents. To the agent $i$ we assign the state $x_{i} \in \mathbb{R}^{n}$ whose dynamics is described by

$$
\dot{x}_{i}(t)=A_{i} x_{i}(t)+u_{i}(t) \quad \forall i \in \mathcal{N}:=\{1, \cdots, N\},
$$

where $u_{i} \in \mathbb{R}^{n}$ is the control input and $A_{i} \in \mathbb{R}^{n \times n}$ is the state matrix.

We suppose that the agents are interconnected through a switching network defined by the following family of graphs $\mathcal{G}^{\sigma(t)}=\left(V, E^{\sigma(t)}, G^{\sigma(t)}\right), \sigma(t) \in \mathcal{I}:=\left\{\sigma_{1}, \ldots, \sigma_{m}\right\}$ where by abuse of notation the node set $V=\left\{x_{1}, \ldots, x_{N}\right\}$ represents the set of agents, the edge set $E^{\sigma(t)}$ indicates the active interactions when using topology $\sigma(t)$, and $G^{\sigma(t)}$ is the weighted adjacency matrix associated with topology $\sigma$, i.e. $G^{\sigma}(t):=\left[g_{i j}^{\sigma(t)}\right]_{i, j \in \mathcal{N}}$ with $g_{i j}^{\sigma(t)} \geq 0$ representing the interconnection strength. We will also use the Laplacian matrix $L^{\sigma(t)}$ whose off-diagonal elements are $-g_{i j}^{\sigma(t)}$ while the diagonal ones are $\sum_{j=1}^{N} g_{i j}^{\sigma(t)}$. Notice that the system can have $m$ different behavior, characterized by $\sigma$, in the sequel we will call these behaviors modes. We also consider that $u_{i}$ is a decentralized state feedback law defined as

$$
u_{i}(t)=-\gamma \sum_{j=1}^{N} g_{i j}^{\sigma(t)}\left(x_{i}-x_{j}\right)
$$

where $\gamma \in \mathbb{R}$ represents the controller's strength. It is noteworthy that $\sigma: \mathbb{R}_{+} \mapsto \mathcal{I}:=\left\{\sigma_{1}, \ldots, \sigma_{m}\right\}$ is a piecewise constant function defining the switching topology signal.

Assumption 1: All the graphs $\mathcal{G}^{\sigma_{k}}, \sigma_{k} \in \mathcal{I}$ are directed and strongly connected (i.e. there exists a path between any two nodes).

Goal: Our objective in this work is to define a switching emergent dynamics and show how well it can approximate the overall behaviour of the large scale network.

\section{Collective Behavior}

Throughout the paper we use $x, u^{\sigma_{k}} \in \mathbb{R}^{n N}$ to denote the vectors collecting the states $x_{i}, i \in \mathcal{N}$ and the controllers $u_{i}^{\sigma_{k}}, i \in \mathcal{N}, \sigma_{k} \in \mathcal{I}$ respectively. With this notation one has

$$
u^{\sigma_{k}}=-\gamma\left(L^{\sigma_{k}} \otimes I_{n}\right) x
$$

and the collective closed-loop dynamics expressed as

$$
\dot{x}(t)=\left(\mathcal{A}-\gamma\left(L^{\sigma_{k}} \otimes I_{n}\right)\right) x(t),
$$

where $\mathcal{A}:=\operatorname{blkdiag}\left(A_{1}, A_{2}, \ldots, A_{N}\right) \in \mathbb{R}^{n N \times n N}$ is the collective block diagonal state matrix. For any $\sigma_{k} \in \mathcal{I}$ let us denote in the following by $\lambda_{\ell}^{\sigma_{k}}, \ell \in\{1, \ldots, N\}$ the eigenvalues of $L^{\sigma_{k}}$ ordered such as $0=\lambda_{1}^{\sigma_{k}}<\Re\left(\lambda_{2}^{\sigma_{k}}\right) \leq \ldots \leq$
$\Re\left(\lambda_{N}^{\sigma_{k}}\right)$. Let us also denote by $v_{l 1}^{\sigma_{k}}$ the left eigenvector of $L^{\sigma_{k}}$ associated with the eigenvalue 0 and whose components sum up to 1 i.e., $v_{l 1}^{\sigma_{k} T} \cdot \mathbf{1}_{N}=1$. It is worth emphasizing that under Assumption 1 one has that $\Re\left(\lambda_{2}^{\sigma_{k}}\right)>0$ and all components of $v_{l 1}^{\sigma_{k}} \in \mathbb{R}^{N}$ are real and strictly positive. In the sequel the left and right eigenvectors of $L^{\sigma_{k}}$ associated with the eigenvalue 0 are given by

$$
v_{l 1}^{\sigma_{k}}=\left[\vartheta_{1}^{\sigma_{k}}, \ldots, \vartheta_{N}^{\sigma_{k}}\right]^{T}, v_{r 1}^{\sigma_{k}}=\mathbf{1}_{N}
$$

Furthermore, there exists a Jordan decomposition of the Laplacian $L^{\sigma_{k}}$, of the form $L^{\sigma_{k}}=U^{\sigma_{k}} \Lambda^{\sigma_{k}} U^{\sigma_{k}-1}$, where $U^{\sigma_{k}} \in \mathbb{R}^{N \times N}$ is non-singular and due to Assumption 1 $\Lambda^{\sigma_{k}} \in \mathbb{C}^{N \times N}$ is the diagonal matrix with the eigenvalues of $L^{\sigma_{k}}$ on the main diagonal. Additionally, the matrix $U^{\sigma_{k}}$ is composed of generalized right eigenvectors of the Laplacian matrix $L^{\sigma_{k}}$ among which the first is $v_{r 1}^{\sigma_{k}}=\mathbf{1}_{N}$. For further purpose, we decompose the matrix $U^{\sigma_{k}}$ as

$$
U^{\sigma_{k}}=\left[\begin{array}{ll}
\mathbf{1}_{N} & V^{\sigma_{k}}
\end{array}\right]
$$

where $V^{\sigma_{k}} \in \mathbb{R}^{N \times(N-1)}$. Similarly, the first row of the $\left(U^{\sigma_{k}}\right)^{-1}$ corresponds to the left eigenvector of $L^{\sigma_{k}}$, i.e.,

$$
\left(U^{\sigma_{k}}\right)^{-1}=\left[\begin{array}{l}
v_{l 1}^{\sigma_{k} T} \\
V^{\sigma_{k} \dagger}
\end{array}\right]
$$

We also define the weighted average of the systems' states $x_{s}$ :

$$
x_{s}=\sum_{i=1}^{N} \vartheta_{i}^{\sigma_{k}} x_{i}, \quad \sum_{i=1}^{N} \vartheta_{i}^{\sigma_{k}}=1 .
$$

Here, we remark that the weighted average term $x_{s}$ is switching with the change in network topology $\sigma_{k}$. In the above network behavior (4), when $N$ is small, it is feasible to study the asymptotic behavior of the agents. However, when the number of agents in the network is large i.e., $N$ is very large, the computational complexity increases and the problem becomes intractable. The main focus of this work lies in developing the reduced order model that approximates the network model (4).

In the following, we consider $\left\{t_{k}\right\}_{k \in \mathbb{N}}$ the unbounded sequence of switching instants. Next, using the modedependent coordinate transformation [13], we express the collective behavior of the network in terms of the mean-field $x_{s}$ and synchronization error $e_{v}$. It is also shown that due to this mode-dependent transformation, impulses are introduced to the dynamics in addition to the switching, leading to a switching-impulsive system. Explicitly, we introduce the following mode-dependent coordinates:

$$
\bar{x}(t):=\left(\mathcal{U}^{\sigma_{k}}\right)^{-1} x(t), \quad t \in\left[t_{k}, t_{k+1}\right)
$$

where $\mathcal{U}^{\sigma_{k}}=\left(U^{\sigma_{k}} \otimes I_{n}\right) \in \mathbb{R}^{n N \times n N}$. Moreover, using equation (7) the collective state of the network in the new coordinates is:

$$
\bar{x}(t)=\left[\begin{array}{l}
\bar{x}_{1}(t) \\
\bar{x}_{2}(t)
\end{array}\right]:=\left[\begin{array}{l}
v_{l 1}^{\sigma_{k} T} \otimes I_{n} \\
V^{\sigma_{k} \dagger} \otimes I_{n}
\end{array}\right] x(t), \quad t \in\left[t_{k}, t_{k+1}\right) .
$$

By remarking that weighted average is defined using the left eigenvectors $v_{l 1}^{\sigma_{k}}$, similarly to (8), the coordinate $\bar{x}_{1}$ represent the weighted average of the systems states, for all $t \in\left[t_{k}, t_{k+1}\right), k \in \mathbb{N}$, as follows 


$$
x_{s}(t)=\left(v_{l 1}^{\sigma_{k} T} \otimes I_{n}\right) x(t),
$$

and the synchronization error is defined as the difference between the individual states and the average $x_{s}$ (see [8])

$$
e(t)=x(t)-\left(\mathbf{1}_{N} \otimes I_{n}\right) x_{s}(t) .
$$

Notice that $\bar{x}_{2}$ is the projection of the synchronization error (11) onto the subspace orthogonal to $v_{l 1}^{\sigma_{k}}$. We also introduce $e_{v} \in \mathbb{R}^{n(N-1)}$ defined $\forall t \in\left[t_{k}, t_{k+1}\right), k \in \mathbb{N}$ as

$$
\begin{aligned}
& e_{v}(t)=\left(V^{\sigma_{k} \dagger} \otimes I_{n}\right) x(t) \\
& =\left(V^{\sigma_{k} \dagger} \otimes I_{n}\right)\left(e(t)+\left(\mathbf{1}_{N} \otimes I_{n}\right) x_{s}(t)\right)=\left(V^{\sigma_{k} \dagger} \otimes I_{n}\right) e(t) .
\end{aligned}
$$

Let us remark that $e(t)=\left(V^{\sigma_{k}} \otimes I_{n}\right) e_{v}(t)$. Although the underlying topology in the original dynamics (4) is only switching, as a consequence of the mode-dependent coordinate transformation (9), jumps are introduced in the new variables $x_{s}$ and $e_{v}$ (see also [14]). The resulting jumps in $x_{s}$ and $e_{v}$, for each $k$ are:

$$
\begin{aligned}
& x_{s}\left(t_{k}\right)=x_{s}\left(t_{k}^{-}\right)+\left(\left(v_{l 1}^{\sigma_{k} T}-v_{l 1}^{\sigma_{k-1} T}\right) \otimes I_{n}\right) x\left(t_{k}\right) \\
& e_{v}\left(t_{k}\right)=e_{v}\left(t_{k}^{-}\right)+\left(\left(V^{\sigma_{k} \dagger}-V^{\sigma_{k-1} \dagger}\right) \otimes I_{n}\right) e\left(t_{k}\right) .
\end{aligned}
$$

Replacing the values of $x\left(t_{k}\right)$ and $e\left(t_{k}\right)$ from equations (11) and (12) along with the properties from (6) and (7), we have,

$$
\begin{aligned}
& x_{s}\left(t_{k}\right)=x_{s}\left(t_{k}^{-}\right)+\left(v_{l 1}^{\sigma_{k} T} V^{\sigma_{k-1}} \otimes I_{n}\right) e_{v}\left(t_{k}^{-}\right) \\
& e_{v}\left(t_{k}\right)=\left(V^{\sigma_{k} \dagger} V^{\sigma_{k-1}} \otimes I_{n}\right) e_{v}\left(t_{k}^{-}\right) .
\end{aligned}
$$

\section{A. Mean-Field Dynamics}

In the new coordinates, $\forall t \in\left[t_{k}, t_{k+1}\right), k \in \mathbb{N}$ we obtain

$$
\begin{aligned}
& \dot{x}_{s}=\left(v_{l 1}^{\sigma_{k} T} \otimes I_{n}\right) \dot{x}=\left(v_{l 1}^{\sigma_{k} T} \otimes I_{n}\right)\left(\mathcal{A}-\gamma\left(L^{\sigma_{k}} \otimes I_{n}\right)\right) x(t) \\
& =\left(v_{l 1}^{\sigma_{k} T} \otimes I_{n}\right)\left(\mathcal{A}-\gamma\left(L^{\sigma_{k}} \otimes I_{n}\right)\right)\left(e+\left(\mathbf{1}_{N} \otimes I_{n}\right) x_{s}\right) \\
& =\left(\left(v_{l 1}^{\sigma_{k} T} \otimes I_{n}\right) \mathcal{A}\left(\mathbf{1}_{N} \otimes I_{n}\right)\right) x_{s}+\left(v_{l 1}^{\sigma_{k} T} \otimes I_{n}\right) \mathcal{A} e
\end{aligned}
$$

and using $e(t)=\left(V^{\sigma_{k}} \otimes I_{n}\right) e_{v}(t)$, we get

$$
\begin{aligned}
\dot{x}_{s} & =\left(\left(v_{l 1}^{\sigma_{k} T} \otimes I_{n}\right) \mathcal{A}\left(\mathbf{1}_{N} \otimes I_{n}\right)\right) x_{s} \\
& +\left(v_{l 1}^{\sigma_{k} T} \otimes I_{n}\right) \mathcal{A}\left(V^{\sigma_{k}} \otimes I_{n}\right) e_{v}
\end{aligned}
$$

representing the mean-field dynamics.

\section{B. Error Dynamics}

Similarly, to obtain the dynamics of the error we start by differentiating on both sides of equation (12) and substituting from (11). For all $t \in\left[t_{k}, t_{k+1}\right), k \in \mathbb{N}$ it follows:

$\dot{e}_{v}=\left(V^{\sigma_{k} \dagger} \otimes I_{n}\right) \dot{e}(t)=\left(V^{\sigma_{k} \dagger} \otimes I_{n}\right)\left(\dot{x}(t)-\left(\mathbf{1}_{N} \otimes I_{n}\right) \dot{x}_{s}(t)\right)$

and replacing the value of $\dot{x}$ and $\dot{x}_{s}$ from equations (4) and (16), respectively, we have the following,

$$
\begin{aligned}
& \dot{e_{v}}=\left(V^{\sigma_{k} \dagger} \otimes I_{n}\right)\left\{\left(\mathcal{A}-\gamma\left(L^{\sigma_{k}} \otimes I_{n}\right)\right)\left(V^{\sigma_{k}} \otimes I_{n}\right) e_{v}(t)\right. \\
& +\mathcal{A}\left(\mathbf{1}_{N} \otimes I_{n}\right) x_{s}(t)-\left(\mathbf{1}_{N} v_{l 1}^{\sigma_{k} T} \otimes I_{n}\right) \mathcal{A}\left(\mathbf{1}_{N} \otimes I_{n}\right) x_{s} \\
& \left.+\left(\mathbf{1}_{N} v_{l 1}^{\sigma_{k} T} \otimes I_{n}\right) \mathcal{A}\left(V^{\sigma_{k}} \otimes I_{n}\right) e_{v}\right\} .
\end{aligned}
$$

Since $V^{\sigma_{k} \dagger} \mathbf{1}_{N}=0$ (see (6) and (7)), it follows that $\dot{e}_{v}=-\gamma\left(V^{\sigma_{k} \dagger} L^{\sigma_{k}} V^{\sigma_{k}} \otimes I_{n}\right) e_{v}+\left(V^{\sigma_{k} \dagger} \otimes I_{n}\right) \mathcal{A}\left(\mathbf{1}_{N} \otimes I_{n}\right) x_{s}$

$$
+\left(V^{\sigma_{k} \dagger} \otimes I_{n}\right) \mathcal{A}\left(V^{\sigma_{k}} \otimes I_{n}\right) e_{v}
$$

and finally, the error dynamics is obtained as follows,

$$
\begin{aligned}
\dot{e}_{v}= & -\gamma\left(\Lambda^{\sigma_{k}^{\prime}} \otimes I_{n}\right) e_{v}+\left(V^{\sigma_{k} \dagger} \otimes I_{n}\right) \mathcal{A}\left(\mathbf{1}_{N} \otimes I_{n}\right) x_{s} \\
& +\left(V^{\sigma_{k} \dagger} \otimes I_{n}\right) \mathcal{A}\left(V^{\sigma_{k}} \otimes I_{n}\right) e_{v}
\end{aligned}
$$

where, $\Lambda^{\sigma_{k}^{\prime}}=\operatorname{diag}\left(\lambda_{2}^{\sigma_{k}} \cdots \lambda_{N}^{\sigma_{k}}\right)$.

Let us write (16) and (18) in a compact form as follows

$$
\left(\begin{array}{l}
\dot{x}_{s}(t) \\
\dot{e}_{v}(t)
\end{array}\right)=\left(\begin{array}{cc}
A_{0}^{\sigma_{k}} & B_{1}^{\sigma_{k}} \\
B_{2}^{\sigma_{k}} & \gamma A_{22}^{\sigma_{k}}+B_{3}^{\sigma_{k}}
\end{array}\right)\left(\begin{array}{l}
x_{s}(t) \\
e_{v}(t)
\end{array}\right),
$$

$\forall t \in\left[t_{k}, t_{k+1}\right), k \in \mathbb{N}$, where for all $\sigma_{k} \in \mathcal{I}$ one has

$$
\begin{aligned}
& A_{0}^{\sigma_{k}}=\left(v_{l 1}^{\sigma_{k} T} \otimes I_{n}\right) \mathcal{A}\left(\mathbf{1}_{N} \otimes I_{n}\right)=\sum_{i=1}^{N} \vartheta_{i}^{\sigma_{k}} A_{i}, \\
& B_{1}^{\sigma_{k}}=\left(v_{l 1}^{\sigma_{k} T} \otimes I_{n}\right) \mathcal{A}\left(V^{\sigma_{k}} \otimes I_{n}\right), \\
& B_{2}^{\sigma_{k}}=\left(V^{\sigma_{k} \dagger} \otimes I_{n}\right) \mathcal{A}\left(\mathbf{1}_{N} \otimes I_{n}\right), \\
& B_{3}^{\sigma_{k}}=\left(V^{\sigma_{k} \dagger} \otimes I_{n}\right) \mathcal{A}\left(V^{\sigma_{k}} \otimes I_{n}\right), A_{22}^{\sigma_{k}}=-\left(\Lambda^{\sigma_{k}^{\prime}} \otimes I_{n}\right) .
\end{aligned}
$$

Property 1: The matrix $A_{22}^{\sigma_{k}}=-\left(\Lambda^{\sigma_{k}^{\prime}} \otimes I_{n}\right)$ is Hurwitz for all $\sigma_{k} \in \mathcal{I}$ since the diagonal matrix $\Lambda^{\sigma_{k}^{\prime}}=$ diag $\left(\lambda_{2}^{\sigma_{k}}, \cdots \lambda_{N}^{\sigma_{k}}\right)$ has all positive eigenvalues.

We highlight that some of the matrices $A_{0}^{\sigma_{k}}, \forall k \in \mathbb{N}$ may not be Hurwitz.

\section{Time-ScAle Separation}

In the sequel we assume that $\gamma$ is a high gain. Let us denote $\epsilon=1 / \gamma$ which is a small parameter since $\gamma$ is large. Dividing both sides of (18) by $\gamma$, we obtain $\forall t \in\left[t_{k}, t_{k+1}\right), k \in \mathbb{N}$

$$
\left(\begin{array}{c}
\dot{x}_{s}(t) \\
\epsilon \dot{e}_{v}(t)
\end{array}\right)=\left(\begin{array}{cc}
A_{0}^{\sigma_{k}} & B_{1}^{\sigma_{k}} \\
\epsilon B_{2}^{\sigma_{k}} & A_{22}^{\sigma_{k}}+\epsilon B_{3}^{\sigma_{k}}
\end{array}\right)\left(\begin{array}{c}
x_{s}(t) \\
e_{v}(t)
\end{array}\right) .
$$

Notice that the $\epsilon$ term multiplied to $\dot{e}_{v}$ is the perturbation parameter, that emphasizes the time-scale separation between the dynamics of $x_{s}$ and $e_{v}$. At the same time, the jumps described by (13)-(14) for the systems are encapsulated in the following impulsive dynamics for the singularly perturbed system (21)

$$
\left(\begin{array}{c}
x_{s}\left(t_{k}\right) \\
e_{v\left(t_{k}\right)}
\end{array}\right)=J^{\sigma_{k-1} \rightarrow \sigma_{k}}\left(\begin{array}{c}
x_{s}\left(t_{k}^{-}\right) \\
e_{v}\left(t_{k}^{-}\right)
\end{array}\right)
$$

where for all $i, i^{\prime} \in \mathcal{I}$,

$$
J^{i \rightarrow i^{\prime}}=\left(\begin{array}{ll}
J_{11}^{i \rightarrow i^{\prime}} & J_{12}^{i \rightarrow i^{\prime}} \\
J_{21}^{i \rightarrow i^{\prime}} & J_{22}^{i \rightarrow i^{\prime}}
\end{array}\right)=\left(\begin{array}{cc}
I_{n} & \left(v_{l 1}^{i^{\prime} T} V^{i} \otimes I_{n}\right) \\
\mathbf{0}_{n(N-1), n} & \left(V^{i^{\prime \dagger}} V^{i} \otimes I_{n}\right)
\end{array}\right) .
$$

Later on, the matrix $J$ will be used to represent the jump on the states of the singularly perturbed system (21) due to the change of network topology.

Hence, the system is written in the standard singular perturbations form with reduced (slow) variable $x_{s}$ and boundary layer (fast) variable $e_{v}$. This time scale separation of the dynamics of networked linear heterogeneous systems is due to the large coupling parameter $\gamma$ and the coordinate transformation (9) which are intrinsic of the network. The most important characteristic of the singularly-perturbed problems is that they can be decomposed into reduced (slow) and boundary-layer (fast) problems, which is dictated by the time-scale separation. 


\section{A. Slow Subsystem}

The slow subsystem is obtained from the original system (21)-(22) by setting $\epsilon=0$. Let us use the notation $x_{e}$ and $e_{s}$ to represent the variable of the original system (21)-(22) in the slow time scale. It is clear from equation (21) that setting $\epsilon=0$ results in $e_{s}=0$ and the slow dynamics is obtained as follows,

$$
\begin{aligned}
\dot{x}_{e}(t) & =A_{0}^{\sigma_{k}} x_{e}(t) \quad \forall t \in\left[t_{k}, t_{k+1}\right), k \in \mathbb{N} \\
x_{e}\left(t_{k}\right) & =x_{e}\left(t_{k}^{-}\right), \quad \forall k \geq 1 .
\end{aligned}
$$

Notice that the jumps does not occurs in the slow dynamics during network switch. Sometimes, the dynamics (23)-(24) is also referred to as emergent dynamics, which is the meanfield dynamics restricted to the synchronization manifold, where $e_{v}=0$.

Remark 1: We note that the emergent dynamics requires the global information of the network and we assume that such information is available at every switching instance.

\section{B. Fast Subsystem}

The behavior of the fast subsystem is obtained by changing the time scale and setting $\epsilon=0$. Let $e_{f}, \tau$ and $x_{f}$ represent the error, time and mean-field in fast time scale. The representation of the fast dynamics assumes that the emergent dynamics $\left(x_{f}\right)$ is constant during the fast transients i.e., $\dot{x}_{f}=0$. As a result, we obtain the fast subsystem as follows,

$$
\begin{aligned}
& \frac{d e_{f}(\tau)}{d \tau}=A_{22}^{\sigma_{k}} e_{f}(\tau), \quad \forall \tau \in\left[\tau_{k}, \tau_{k+1}\right), \forall k \in \mathbb{N} \\
& e_{f}\left(\tau_{k}\right)=J_{22}^{\sigma_{k-1} \rightarrow \sigma_{k}} e_{f}\left(\tau_{k}^{-}\right),
\end{aligned}
$$

where, we recall that $J_{22}^{\sigma_{k-1} \rightarrow \sigma_{k}}=\left(V^{\sigma_{k} \dagger} V^{\sigma_{k-1}} \otimes I_{n}\right)$ and $\tau_{k}=t_{k} / \epsilon$. We know from Property 1 , the matrix $A_{22}^{\sigma_{k}}, \forall k \in \mathbb{N}$ is Hurwitz. This fast subsystem is usually called the boundary-layer dynamics [4].

\section{ApProximate Models}

In order to prove that the switching emergent dynamics (23)-(24) provides a good dynamic approximation for the average behaviour of the overall behavior of the closedloop dynamics (4) we adapt the results in [4]. In contrast to [14], where all the slow and fast subsystems are assumed to be asymptotically stable, here it is assumed that the slow subsystems are not necessarily all asymptotically stable. Therefore, we next introduce a dwell-time condition between two consecutive switching in order to guarantee the boundedness of the emergent dynamics trajectory. To this aim we first recall the following definition of the measure of a square matrix [3].

Definition 1: The measure of a square matrix $M$ is defined as $\nu(M)=\frac{1}{2} \lambda_{\max }\left(M+M^{T}\right)$, where $\lambda_{\max }$ is the largest eigenvalue of the symmetric matrix $M+M^{T}$.

Lemma 1 ([3]): For any square matrix $\mathbb{A}$ the following holds:

$$
\left\|e^{\mathbb{A} t}\right\| \leq e^{\nu(\mathbb{A}) t}
$$

Then from the definition of the measure and from equation (20) for the state matrices of the slow dynamics, we have,

$$
\nu\left(A_{0}^{\sigma_{k}}\right)=\frac{1}{2} \lambda_{\max }^{\sigma_{k}}\left(A_{0}^{\sigma_{k}}+A_{0}^{\sigma_{k} T}\right), \quad \forall \sigma_{k} \in \mathcal{I} .
$$

We notice that $\nu\left(A_{0}^{\sigma_{k}}\right)$ may be positive or negative. Let $\widetilde{S}$ and $\widetilde{U}$ be the set of stable and unstable modes, respectively. We will say that during the interval $\left[t_{k}, t_{k+1}\right)$, the system is in the stable mode (i.e., $\left.\sigma_{k} \in \widetilde{S}\right)$ iff $\nu\left(A_{0}^{\sigma_{k}}\right)<0$ and the system is in unstable mode $\left(\sigma_{k} \in \widetilde{U}\right)$ iff $\nu\left(A_{0}^{\sigma_{k}}\right) \geq 0$. Moreover, $\widetilde{S} \cup \widetilde{U}=\mathcal{I}$.

\section{A. Boundedness of Emergent Dynamics}

Let $\delta_{k}=\left(t_{k+1}-t_{k}\right)$ be the time spent in mode $\sigma_{k}$ for any $k \in \mathbb{N}$. For any $t \in \mathbb{R}_{+}$we define $t_{s}(t)=\bigcup_{k, \sigma_{k} \in \widetilde{S}} \delta_{k}$ and $t_{u}(t)=\bigcup_{k, \sigma_{k} \in \widetilde{U}} \delta_{k}$ be the total amount of time before $t$ the system remains in the stable and unstable modes, respectively and $\left[t_{0}, t\right)=t_{s}(t) \bigcup t_{u}(t)$ where $t_{0}$ is the initial time. Also, we define the following,

$$
\lambda_{s}=\max _{\sigma_{k} \in \widetilde{S}} \nu\left(A_{0}^{\sigma_{k}}\right)<0, \quad \lambda_{u}=\max _{\sigma_{k} \in \widetilde{U}} \nu\left(A_{0}^{\sigma_{k}}\right)>0 .
$$

Lemma 2: The trajectory $x_{e}\left(t, x_{e}\left(t_{0}\right)\right)$ of the slow subsystem (23)-(24) is bounded for all $t \in \mathbb{R}_{+}$and all $x_{e}\left(t_{0}\right) \in \mathbb{R}^{n}$ if $\exists t^{*}>0$ such that

$$
\frac{t_{s}(t)}{t_{u}(t)} \geq-\frac{\lambda_{u}}{\lambda_{s}}, \quad \forall t>t^{*} .
$$

Remark 2: Inequality (29) can be considered as a stabilizing condition for the slow dynamics (23)-(24).

Remark 3: It is easy to show that if all the modes are at least marginally stable, then the trajectories of the slow subsystem will be bounded as well.

Proof: Let $\left\{t_{k}\right\}_{k \in \mathbb{N}}$ be the switching time for the switching event $\sigma_{k}$. Then for any $t \in\left[t_{k}, t_{k+1}\right)$, from equations (23)(24) we can express $x_{e}(t)$ as a function of $x_{e}\left(t_{k-1}\right)$ as,

$$
x_{e}(t)=e^{A_{0}^{\sigma_{k}}\left(t-t_{k}\right)} e^{A_{0}^{\sigma_{k-1}}\left(t_{k}-t_{k-1}\right)} x_{e}\left(t_{k-1}\right) .
$$

Recursively, we have

$$
x_{e}(t)=e^{A_{0}^{\sigma_{k}}\left(t-t_{k}\right)} e^{A_{0}^{\sigma_{k-1}}\left(t_{k}-t_{k-1}\right)} \cdots e^{A_{0}^{\sigma_{0}}\left(t_{0}-t_{1}\right)} x_{e}\left(t_{0}\right) .
$$

Then from Lemma 1 and equation (30), with $\delta_{k}=\left(t_{k+1}-\right.$ $\left.t_{k}\right), k \in \mathbb{N}$ we get,

$$
\begin{aligned}
& \left\|x_{e}(t)\right\|=\left\|e^{A_{0}^{\sigma_{k}}\left(t-t_{k}\right)} \cdots e^{A_{0}^{\sigma_{0}}\left(t_{1}-t_{0}\right)} x_{e}\left(t_{0}\right)\right\| \\
& \leq\left\|e^{A_{0}^{\sigma_{k}}\left(t-t_{k}\right)}\right\| \cdots\left\|e^{A_{0}^{\sigma_{0}}\left(t_{1}-t_{0}\right)}\right\|\left\|x_{e}\left(t_{0}\right)\right\| \\
& \leq e^{\nu\left(A_{0}^{\sigma_{k}}\right)\left(t-t_{k}\right)+\cdots+\nu\left(A_{0}^{\sigma_{0}}\right)\left(t_{1}-t_{0}\right)}\left\|x_{e}\left(t_{0}\right)\right\| \\
& =e^{\left(\sum_{\sigma_{j} \in \widetilde{U}} \nu\left(A_{0}^{\sigma_{j}}\right) \delta_{j}\right)+\left(\sum_{\sigma_{j} \in \widetilde{S}} \nu\left(A_{0}^{\sigma_{j}}\right) \delta_{j}\right)}\left\|x_{e}\left(t_{0}\right)\right\| \\
& \leq e^{\lambda_{u} t_{u}+\lambda_{s} t_{s}}\left\|x_{e}\left(t_{0}\right)\right\| .
\end{aligned}
$$

Using (29) we conclude the proof.

\section{B. Stability of the Fast Dynamics}

Since we know that all the fast modes are stable (see Property 1 ), the measure $\nu\left(A_{22}^{\sigma_{k}}\right)<0$, for all $k \in \mathbb{N}$. Next we introduce

$$
\begin{gathered}
\lambda_{f}:=-\max _{\sigma_{k} \in \mathcal{I}} \nu\left(A_{22}^{\sigma_{k}}\right)>0 \\
\gamma_{22}=\max _{\sigma_{k}, \sigma_{k-1} \in \mathcal{I},}\left\|J_{22}^{\sigma_{k-1} \rightarrow \sigma_{k}}\right\| .
\end{gathered}
$$

Lemma 3: Consider the fast dynamics (25)-(26) with initial condition $e_{f}\left(t_{0} / \epsilon\right) \in \mathbb{R}^{n(N-1)}$ and switching time 
sequences $\left\{t_{k}\right\}_{k \geq 0}$ satisfying the dwell time condition $0<$ $\tau^{*} \leq \delta_{k}, \forall k \in \mathbb{N}$. Then the following conditions holds:

1) if $\gamma_{22} \leq 1$ then the fast dynamics is exponentially stable for any $\tau^{*}>0$ and

2) if $\gamma_{22}>1$ then the fast dynamics is exponentially stable for any $\tau^{*}$ satisfying the following,

$$
\tau^{*}>\frac{\epsilon \ln \left(\gamma_{22}\right)}{\lambda_{f}}
$$

Proof: Using the same idea from Lemma (2) and integrating (25) - (26) one has that

$$
e_{f}(\tau)=e^{A_{22}^{\sigma_{k}}\left(\frac{t-t_{k}}{\epsilon}\right)} J_{22}^{\sigma_{k-1} \rightarrow \sigma_{k}} e^{A_{22}^{\sigma_{k-1}}\left(\frac{t_{k}-t_{k-1}}{\epsilon}\right)} e_{f}\left(\frac{t_{k-1}}{\epsilon}\right),
$$

$\forall \tau \in\left[t_{k} / \epsilon, t_{k+1} / \epsilon\right), k \in \mathbb{N}$. This recursively yields

$e_{f}(\tau)=e^{A_{0}^{\sigma_{k}}\left(\frac{t-t_{k}}{\epsilon}\right)} J_{22}^{\sigma_{k-1} \rightarrow \sigma_{k}} \cdots J_{22}^{\sigma_{0} \rightarrow \sigma_{1}} e^{A_{0}^{\sigma_{0}}\left(\frac{t_{1}-t_{0}}{\epsilon}\right)} e_{f}\left(\frac{t_{0}}{\epsilon}\right)$.

Let $\tau^{*}>0$ such that $\tau^{*} \leq\left(t_{k+1}-t_{k}\right), \forall k \in \mathbb{N}$ then we have the following

$\left\|e_{f}(\tau)\right\|=\left\|e^{A_{0}^{\sigma_{k}}\left(\frac{\tau^{*}}{\epsilon}\right)} J_{22}^{\sigma_{k-1} \rightarrow \sigma_{k}} \cdots J_{22}^{\sigma_{0} \rightarrow \sigma_{1}} e^{A_{0}^{\sigma_{0}}\left(\frac{\tau^{*}}{\epsilon}\right)} e_{f}\left(\frac{t_{0}}{\epsilon}\right)\right\|$
$\leq \gamma_{22}^{k} e^{\nu\left(A_{22}^{\sigma_{k}}\right) \frac{\tau^{*}}{\epsilon}+\nu\left(A_{22}^{\sigma_{k-1}}\right) \frac{\tau^{*}}{\epsilon}+\cdots+\nu\left(A_{22}^{\sigma_{0}}\right) \frac{\tau^{*}}{\epsilon}}\left\|e_{f}\left(\frac{t_{0}}{\epsilon}\right)\right\|$
$\leq \gamma_{22}^{k} e^{-\lambda_{f}(k+1) \frac{\tau^{*}}{\epsilon}}\left\|e_{f}\left(\frac{t_{0}}{\epsilon}\right)\right\|=e^{k \ln \gamma_{22}} e^{-\lambda_{f}(k+1) \frac{\tau^{*}}{\epsilon}}\left\|e_{f}\left(\frac{t_{0}}{\epsilon}\right)\right\|$

If $\gamma_{22} \leq 1$, it is clear from (36) that condition 1) holds. When $\gamma_{22}>1$, continuing from equation (36), we have

$$
\begin{aligned}
& \left\|e_{f}(\tau)\right\| \leq e^{-\ln \left(\gamma_{22}\right)+(k+1) \ln \left(\gamma_{22}\right)} e^{-\lambda_{f}(k+1) \frac{\tau^{*}}{\epsilon}}\left\|e_{f}\left(t_{0} / \epsilon\right)\right\| \\
& =e^{-\ln \left(\gamma_{22}\right)} e^{(k+1)\left(\ln \left(\gamma_{22}\right)-\lambda_{f} \frac{\tau^{*}}{\epsilon}\right)}\left\|e_{f}\left(t_{0} / \epsilon\right)\right\| .
\end{aligned}
$$

Consequently (37) ensures the fast subsystem is exponentially stable if $\left(\ln \left(\gamma_{22}\right)-\lambda_{f} \frac{\tau^{*}}{\epsilon}\right)<0$ and using (33) we conclude that condition 2) holds.

\section{Closeness of the Approximate Model}

We are now ready to prove the slow model (23)-(24) and the fast model (25)-(26) provide a good approximation of the overall dynamics (21)-(22) under the stability conditions proposed in Lemmas (2) and (3). The next result gives the approximation of synchronization error dynamics $e_{v}$ of the original system in terms of the synchronization error $e_{f}$ for the fast dynamics and the parameter $\epsilon$.

Proposition 1: For any $t \geq t_{0}$ the following approximation holds true

$$
e_{v}(t)=e_{f}(\tau)+\mathcal{O}(\epsilon), \quad \tau=\frac{t}{\epsilon} .
$$

Proof: Remarking that the fast dynamics are asymptotically stable for each mode, before the first switch $t_{1}$, the following approximation holds

$$
e_{v}(t)=e_{f}(\tau)+\mathcal{O}(\epsilon), \quad \forall t \in\left[t_{0}, t_{1}\right) .
$$

Also, we know for any $t \in\left[t_{0}, t_{1}\right)$, the fast dynamics is

$$
e_{f}(\tau)=e_{f}(t / \epsilon)=e^{A_{22}^{\sigma_{0}}\left(\frac{t-t_{0}}{\epsilon}\right)} e_{f}(t / \epsilon)
$$

and from (39) and (40), one gets

$$
e_{v}(t)=e^{A_{22}^{\sigma_{0}}\left(\frac{t-t_{0}}{\epsilon}\right)} e_{f}\left(t_{0} / \epsilon\right)+\mathcal{O}(\epsilon) \text {. }
$$

From Lemma (3), for all $t \in\left[t_{1}, t_{2}\right)$, we obtain,

$$
e_{v}(t)=e^{A_{22}^{\sigma_{1}}\left(\frac{t-t_{1}}{\epsilon}\right)} J_{22}^{\sigma_{0} \rightarrow \sigma_{1}}\left(e^{A_{22}^{\sigma_{0}}\left(\frac{t_{1}-t_{0}}{\epsilon}\right)} e_{f}\left(\frac{t_{0}}{\epsilon}\right)+\mathcal{O}(\epsilon)\right)
$$

Continuing the reasoning, for any $k$, for all $t \in\left[t_{k}, t_{k+1}\right)$ one has

$$
\begin{aligned}
& e_{v}(t)=e^{A_{22}^{\sigma_{k}}\left(\frac{t-t_{k}}{\epsilon}\right)} J_{22}^{\sigma_{k-1} \rightarrow \sigma_{k}} \ldots J_{22}^{\sigma_{0} \rightarrow \sigma_{1}} e^{A_{22}^{\sigma_{0}}\left(\frac{t_{1}-t_{0}}{\epsilon}\right)} e_{f}\left(\frac{t_{0}}{\epsilon}\right) \\
& +e^{A_{22}^{\sigma_{k}}\left(\frac{t-t_{k}}{\epsilon}\right)} J_{22}^{\sigma_{k-1} \rightarrow \sigma_{k}} \ldots e^{A_{22}^{\sigma_{1}}\left(\frac{t-t_{1}}{\epsilon}\right)} J_{22}^{\sigma_{0} \rightarrow \sigma_{1}} \mathcal{O}(\epsilon)
\end{aligned}
$$

We show that the second term in (42) is of order $\mathcal{O}(\epsilon)$. Assuming that the switching-impulsive systems satisfy the dwell-time condition (33) from (31), (32) we establish the upper-bound on the second term of (42) as follows

$$
\begin{aligned}
& \left\|e^{A_{22}^{\sigma_{k}}\left(\frac{t-t_{k}}{\epsilon}\right)} J_{22}^{\sigma_{k-1} \rightarrow \sigma_{k}} \ldots e^{A_{22}^{\sigma_{1}}\left(\frac{t_{2}-t_{1}}{\epsilon}\right)} J_{22}^{\sigma_{0} \rightarrow \sigma_{1}}\right\| \\
& \leq\left\|e^{A_{22}^{\sigma_{k}}\left(\frac{t-t_{k}}{\epsilon}\right)}\right\|\left\|J_{22}^{\sigma_{k-1} \rightarrow \sigma_{k}}\right\| \ldots\left\|e^{A_{22}^{\sigma_{1}}\left(\frac{t_{2}-t_{1}}{\epsilon}\right)}\right\|\left\|J_{22}^{\sigma_{0} \rightarrow \sigma_{1}}\right\| \\
& \leq \gamma_{22}^{k} e^{\left(\nu\left(A_{22}^{\sigma_{k}}\right)+\ldots+\nu\left(A_{22}^{\sigma_{1}}\right)\right)\left(\frac{\tau^{*}}{\epsilon}\right)} \leq \gamma_{22}^{k} e^{-\lambda_{f} k\left(\frac{t-t_{1}}{\epsilon}\right)} \\
& =e^{k \ln \left(\gamma_{22}\right)-\lambda_{f} k\left(\frac{\tau^{*}}{\epsilon}\right)}=e^{k\left(\ln \left(\gamma_{22}\right)-\lambda_{f} \frac{\tau^{*}}{\epsilon}\right)}<1 .
\end{aligned}
$$

It is clear from (43) that the second term from (42) is bounded and tends to zero as $\mathrm{t} \rightarrow \infty$, when the dwelltime condition (33) is satisfied. Consequently, (38) holds meaning that, the error is approximated by the boundarylayer state and it is stable when the dwell-time condition (33) is satisfied.

It is noteworthy that the error in the slow time scale $e_{s}(t)$ is zero and hence the overall error dynamics $e_{v}(t)$ is approximated by the error in the fast time scale $e_{f}(\tau)$ only. The next result provides the approximation of the mean-field dynamics $x_{s}(t)$ in term of the emergent dynamics $x_{e}(t)$.

Proposition 2: Under Assumption (1), if the trajectory of the emergent dynamics is bounded then the dynamics of the original systems (21)-(22) is approximated for time $t \geq t_{0}$ by emergent dynamics (23)-(24) with the order of approximation $\mathcal{O}(\epsilon)$, i.e.,

$$
x_{s}(t)=x_{e}(t)+\mathcal{O}(\epsilon) .
$$

Proof: For the continuous dynamics, we know from [4, Theorem 5.1], the slow subsystem approximates the original system on the finite time interval i.e., before the switching event $\sigma_{1}$, as follows,

$$
x_{s}(t)=x_{e}(t)+\mathcal{O}(\epsilon) \quad \forall t \in\left[t_{0}, t_{1}\right),
$$

and following from (23)-(24) the slow subsystem (emergent dynamics) is

$$
x_{e}(t)=e^{A_{0}^{\sigma_{0}}\left(t-t_{0}\right)} x_{0} \quad \forall t \in\left[t_{0}, t_{1}\right) .
$$

Then, from equations (45) and (46), the mean-field dynamics for all $t \in\left[t_{0}, t_{1}\right)$ is

$$
x_{s}(t)=e^{A_{0}^{\sigma_{0}}\left(t-t_{0}\right)} x_{0}+\mathcal{O}(\epsilon) .
$$

Hence, from the jump matrix (13) and (47), the mean-field dynamics for all $t \in\left[t_{1}, t_{2}\right)$ is

$$
\begin{aligned}
x_{s}(t) & =e^{A_{0}^{\sigma_{1}}\left(t-t_{1}\right)}\left(v_{l 1}^{\sigma_{k} T} V^{\sigma_{k-1}} \otimes I_{n}\right) e_{v}\left(t_{1}\right) \\
& +e^{A_{0}^{\sigma_{1}}\left(t-t_{1}\right)} e^{A_{0}^{\sigma_{0}}\left(t_{1}-t_{0}\right)} x_{0}+e^{A_{0}^{\sigma_{1}}\left(t-t_{1}\right)} \mathcal{O}(\epsilon) .
\end{aligned}
$$

Since the error dynamics is exponentially stable for each mode and $e_{v}(t) \rightarrow 0$, the first term from equation (48) goes to zero for sufficiently large $t_{1}$ and we obtain the following

$$
\begin{aligned}
& x_{s}(t)=e^{A_{0}^{\sigma_{1}}\left(t-t_{1}\right)} e^{A_{0}^{\sigma_{0}}\left(t_{1}-t_{0}\right)} x_{0}+e^{A_{0}^{\sigma_{1}}\left(t-t_{1}\right)} \mathcal{O}(\epsilon) \\
& x_{s}(t)=e^{A_{0}^{\sigma_{1}}\left(t-t_{1}\right)} x_{s}\left(t_{1}\right)+e^{A_{0}^{\sigma_{1}}\left(t-t_{1}\right)} \mathcal{O}(\epsilon) \quad \forall t \in\left[t_{1}, t_{2}\right) .
\end{aligned}
$$


Now, by induction, for any $k$, and for all $t \in\left[t_{k}, t_{k+1}\right)$

$$
\begin{aligned}
x_{s}(t) & =e^{A_{0}^{\sigma_{k}}\left(t-t_{k}\right)} \cdots e^{A_{0}^{\sigma_{1}}\left(t_{2}-t_{1}\right)} e^{A_{0}^{\sigma_{0}}\left(t_{1}-t_{0}\right)} x_{0} \\
& +e^{\left(A_{0}^{\sigma_{k}}\left(t-t_{k}\right)+\ldots+A_{0}^{\sigma_{1}}\left(t-t_{1}\right)\right)} \mathcal{O}(\epsilon)
\end{aligned}
$$

When the condition for the boundedness of the trajectories i.e., the inequality (29) is satisfied, the propagation of $\mathcal{O}(\epsilon)$ as $t \rightarrow \infty$ holds and we have from (49), $\forall t \in\left[t_{k}, t_{k+1}\right)$

$$
\begin{aligned}
& x_{s}(t)=e^{A_{0}^{\sigma_{k}}\left(t-t_{k}\right)} \cdots e^{A_{0}^{\sigma_{1}}\left(t_{2}-t_{1}\right)} e^{A_{0}^{\sigma_{0}}\left(t_{1}-t_{0}\right)} x_{0}+\mathcal{O}(\epsilon) \\
& x_{s}(t)=e^{A_{0}^{\sigma_{k}}\left(t-t_{k}\right)} e\left(t_{k}\right)+\mathcal{O}(\epsilon)=x_{e}(t)+\mathcal{O}(\epsilon),
\end{aligned}
$$

under the assumption of the Lemma (2). This proves that the emergent dynamics, which is the weighted average of the systems states, approximate the mean-field dynamics with $\mathcal{O}(\epsilon)$ order of approximation. Also, it is important to that the asymptotic stability of the fast dynamics is necessary for the $\mathcal{O}(\epsilon)$ approximation of the original variables.

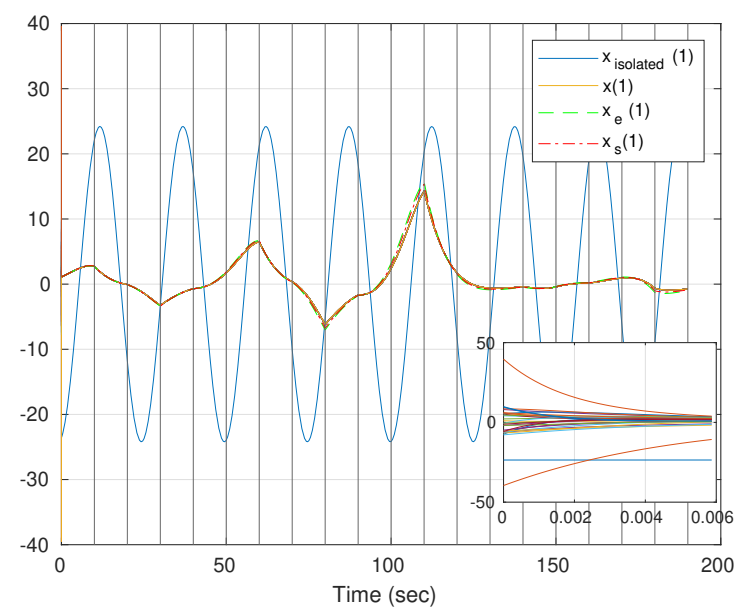

Fig. 1: State trajectories of the collective $(x)$, emergent $\left(x_{e}\right)$ and mean-field dynamics $\left(x_{s}\right)$, dynamics of one of the nodes in the absence of connections, vertical lines represent the switches.

\section{Numerical Results}

In this section, to numerically illustrate our results we consider a network of 30 agents, their dynamics is given by (1) and the state matrices are randomly generated 2D rotational matrices. The initial conditions are also randomly generated in the interval $[-50,50]$. To illustrate typical behaviour of the nodes we plotted one of them in the absence of interconnections. The network structure is considered to be randomly switching between two directed and strongly connected topologies $\mathcal{G}_{1}$ and $\mathcal{G}_{2}$. They are chosen so that $\mathcal{G}_{1}$ results in stable and $\mathcal{G}_{2}$ results in unstable emergent dynamics; the interconnection strength $\gamma=100$ (i.e., $\epsilon=0.01$ ). The dwell time $\tau^{*}=10$ is chosen to satisfy boundedness conditions (29). Simulation results are presented in the Fig.1, where after a short transient that is zoomed in the subplot, the trajectories evolve in (practical) consensus following slow network dynamics and clearly the reduced order state $x_{e}$ approximates the original state $x$. In the subplot, we presented all agents trajectories during the initial interval of 0.006 seconds to make clear the action of the boundary layer dynamics that quickly forces the trajectories to the slow dynamics. Notice that the blue trajectory corresponds to the isolated node, it appears to be constant due to the difference of time scales.

\section{CONCLUSIONS}

In this paper, the synchronizing behavior of linear heterogeneous systems over switching topology is approximated by a reduced ordered system. Using the mode-dependent coordinate transformation, the network is transformed into singular perturbation form and then the reduced-ordered model is obtained using the time-scale separation. A dwelltime conditions between two consecutive switching is established to ensure the boundedness of the solution of the reduced-ordered model and it is proven that this reduced model approximates the collective dynamics.

\section{REFERENCES}

[1] E. Biyık and M. Arcak. Area aggregation and time-scale modeling for sparse nonlinear networks. Sys. \& Ctrl. Letters, 57(2):142-149, 2007.

[2] J.H. Chow and P. Kokotović. Time scale modeling of sparse dynamic networks. IEEE Trans. on Automatic Ctrl, 30(8):714-722, 1985.

[3] M. Jungers, G. S. Deaecto, and J. Geromel. Bounds for the remainders of uncertain matrix exponential and sampled-data control of polytopic linear systems. Automatica, 82:202-208, 2017.

[4] P. Kokotović, H.K. Khalil, and J. O'Reilly. Singular perturbation methods in control: analysis and design. SIAM Series in Classics and Applied Mathematics, 1999.

[5] J.G. Lee and H. Shim. A tool for analysis and synthesis of heterogeneous multi-agent systems under rank-deficient coupling. Automatica, 117:108952, 2020.

[6] Q. Liang, Z. She, L. Wang, M. Chen, and Q.-G. Wang. Characterizations and criteria for synchronization of heterogeneous networks to linear subspaces. SIAM Journal on Control and Optimization, 55, 12 2017.

[7] T. Liu, D. J. Hill, and J. Zhao. Output synchronization of dynamical networks with incrementally-dissipative nodes and switching topology. IEEE Transactions on Circuits and Systems I, 62(9):2312-2323, 2015.

[8] M. Maghenem, E. Panteley, and A. Loria. Singular-perturbations-based analysis of synchronization in heterogeneous networks: A case-study. In IEEE CDC 2016, pages 2581-2586, 2016.

[9] S. Martin, I. C. Morărescu, and D. Nešić. Time scale modeling for consensus in sparse directed networks with time-varying topologies. In IEEE CDC 2016, pages pp. 7-12, 2016.

[10] Z. Meng, T. Yang, D. V. Dimarogonas, and K. H. Johansson. Coordinated output regulation of heterogeneous linear systems under switching topologies. Automatica, 53:362-368, 2015.

[11] J.M. Montenbruck, M. Bürger, and F. Allgöwer. Practical synchronization with diffusive couplings. Automatica, 53:235 - 243, 2015.

[12] I.-C. Morărescu and A. Girard. Opinion dynamics with decaying confidence: Application to community detection in graphs. IEEE Trans. on Automatic Control, 56(8):1862 - 1873, 2011.

[13] E. Panteley and A. Loria. Synchronization and dynamic consensus of heterogeneous networked systems. IEEE Transactions on Automatic Control, 62(8):3758-3773, 2017.

[14] J. Ben Rejeb, I.-C. Morărescu, A. Girard, and J. Daafouz. Stability analysis of a general class of singularly perturbed linear hybrid systems. Automatica, 90:98-108, 2018.

[15] S. H. Strogatz. Sync: The Emerging Science of Spontaneous Order. Hyperion Press, 2003.

[16] L. Wang, M.Z.Q. Chen, and Q.-G. Wang. Bounded synchronization of a heterogeneous complex switched network. Automatica, 56:19 24, 2015.

[17] Peter Wieland, Rodolphe Sepulchre, and Frank Allgöwer. An internal model principle is necessary and sufficient for linear output synchronization. Automatica, 47:1068-1074, 052011. 